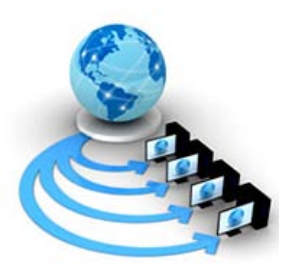

\title{
A WAVELET APPROACH FOR MEDICAL IMAGE DENOISING
}

\author{
Gagandeep Kaur \\ ECE ,Faculty of Engineering \& Technology \\ Manav Rachna International University \\ Faridabad,India
}

\author{
Romika Choudhary \\ ECE ,Faculty of Engineering \& Technology \\ Manav Rachna International University \\ Faridabad,India
}

\author{
Ashish Vats \\ ECE, Faculty of Engineering \& Technology \\ Manav Rachna International University \\ Faridabad,India
}

\begin{abstract}
Medical Images have always been vulnerable to high level components of noises. Magnetic Resonance Imaging (MRI), X-ray, Computed Tomography and Ultrasound are among most popular techniques for producing medical images, during image capture and transmission noise is added in the images that decreases the image quality and leads to poor image analysis. Various denoising techniques are used to remove the noise or distortion from images while preserving the original quality of the image among which wavelet transform has been proved an efficient one in reducing the noise level. The aim of this paper to characterize the Gaussian noise in wavelet transforms subsequently a threshold based denoising algorithm has been developed using hard and soft thresholding techniques that works on Haar, Daubechies and Symlet Transforms. Firstly the image is decomposed using Haar and Daubechies and symlet transforms, and then the level of soft and hard threshold is selected for reducing the noise in the image and finally the comparison between them has been done on the basis of calculated PSNR\& MSE of an image for every wavelet.
\end{abstract}

Keywords: Daubechies, Haar, Symlet, Thresholding MSE, PSNR

\section{INTRODUCTION}

The image is an important part of information carrying system. The noise which corrupts the image interacts with the image during its acquisition or transmission and other causes include hardware faults in the camera lens, lesser processing power etc. which creates undesired elements in image, not soothing to the human eye therefore denoising becomes one of the fundamental problems faced in the field of image processing, Image denoising is an emerging field in the image processing domain so as to remove the noise in the image along with preservation of image details. In the last decade or so the image denoising has acquired tremendous research attraction specially in medical field, satellite imaging and in forensic laboratory images to produce more and more fine and clear pictures as per the requirement of application The main aim in denoising is to obtain the lost elements in the image due to noise without compromising the details of image. In the recent years image denoising using different techniques for wavelet packet has developed as an option to the formerly used filtering and Fourier Transform approaches due to its excellent localization property as well as some compact energy packet reduction. So, it became one of the most reliable and efficient methods compared to others.

\section{RELATED WORK}

During the last decade Donoho and Johnstone [1] pioneered significance of threshold levels and coined their type (i.e. hard or soft threshold). Mallat S.[2] is the developer in demonstrating the use of wavelet transform for non stationary signals. Other approach in wavelet denoising is the use of wavelet transform scale correlation between the wavelet coefficients[3]. In recent years research in wavelet transform has been used in important areas like in aerial images captures by airplane, satellite imaging, SONAR images and medical images

Noise can be catogrized into two types[4] i.e additive noise and multiplicative noise based on the mathematical nature and behavior of the noise. The additive noise adds with the pixel intensity of image whereas the multiplicative noise is in form of multiplication with the image pixels. In the image denoising process, the nature of the noise plays a significant role in denoising. The additive noise is given by, $\mathrm{i}(\mathrm{a}, \mathrm{b})=\mathrm{s}(\mathrm{a}, \mathrm{b})+\mathrm{a}(\mathrm{a}, \mathrm{b}) .$. (1) The multiplicative noise can be presented mathematically as $i(a, b)=s(a, b) \times a(a, b) . .(2)$ where, $i(a, b)$ is the basic information signal, $s(a, b)$ is the noise entered into the original signal which gives out the corrupted noisy image $\mathrm{a}(\mathrm{a}, \mathrm{b})$. (a, b) represents the coordinates of the pixel.

Image denoising has become a significant step in processing of images for removing unwanted noisy elements from the image which enter in the image during various phases of image acquisition and transmission. The image denoising algorithms have to remove the unwanted noisy elements and keep all the important features of the image The image denoising algorithms have to maintain a balance between the two parameters i.e. noise removal and preservation of image features. There are various noise models that correspond to a probability density function which represents the distribution of noise in the image. Images used for some crucial applications have to be noise free for accurate interpretation[5]. Medical image denoising algorithms have attracted much attention with the use of advanced medical equipments. Noise free medical images are desired by a surgeon to assist manual and computer based diagnosis. Selection of appropriate approach is a tough task which is solved with the development of Wavelet Transform. 


\section{WAVELET TRANSFORM}

The energy transformation property of wavelet transform plays an important role in denoising. by which most of the signal energy is contained in a few large wavelet coefficients which quantizies energy and a small portion of the energy is spread across a big number of little wavelet coefficients[6]. The detail coefficients are merged with high frequency noise. By recursive thresholding these detail wavelet coefficients, the image denoising is accomplished while preserving features of the image like sparseness, clustering, and correlation between adjacent wavelet coefficients. Image can be decomposed into several components based on the frequency characteristics of the signal with the help of wavelet transform. Wavelet transform converts the signal information the frequency showing coefficients which are distributed in horizontal, vertical and diagonal parts of the image. These are known as the decomposed frequency components of the under observation image[7]. Another advantage of wavelet transform is multi resolution analysis performed by wavelets Wavelets are reliable in showing efficient results for $t$ localized details like edges, boundaries and curves.The original image is decomposed into four elements namely LL, HL, LH, and $\mathrm{HH}$ by application of horizontal and vertical filters as shown in the Fig. 1 . The LL sub-band gives the approximation or average of the original image. The other three sub-bands are details representing elements of wavelet coefficients namely the vertical sub-bands, diagonal sub-bands and horizontal subbands respectively. The sub bands HL1, HH1 and LH1 represent details rcoefficients while sub-band LL1 demonstrates low level coefficients [8][9]. 2D decomposition is achieved in wavelet transform by further decomposition of LL1 sub band as shown in Fig.2. After decomposition it undergoes wavelet thresholding which will select and parse selected wavelet coefficients, the basic types of thresholding are hard and soft thresholding, where each wavelet coefficient will set to zero for smaller amplitude compared to predefined threshold otherwise it is remained unchanged in hard thresholding while shrunk in an absolute value rather than zero in soft thresholding[10]. Finally the inverse wavelet transform is performed to obtain the reconstructed image.

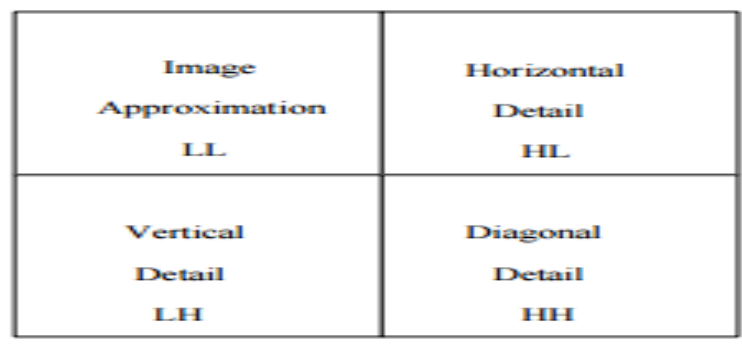

Figure 1: Decomposition of signal using wavelet transform

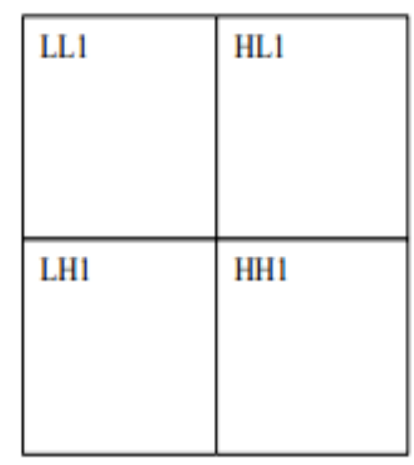

(a)

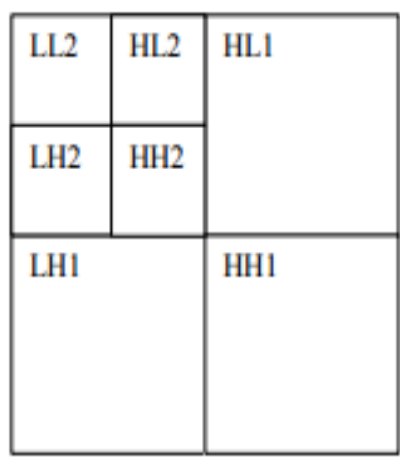

(b)
Figure 2(a) 1-D decomposition (b) 2-D decomposition

\section{METHODOLOGY}

In medical images many wavelets like $\mathrm{db} 1$, sym8, coif1, coif3 etc can be used for denoising, we have used haar, sym and daubechies at certain level of hard and soft threshold for decomposition of noisy images and reconstruction the denoised images. PSNR and MSE of denoised images are calculated for comparison of results of different wavelets.

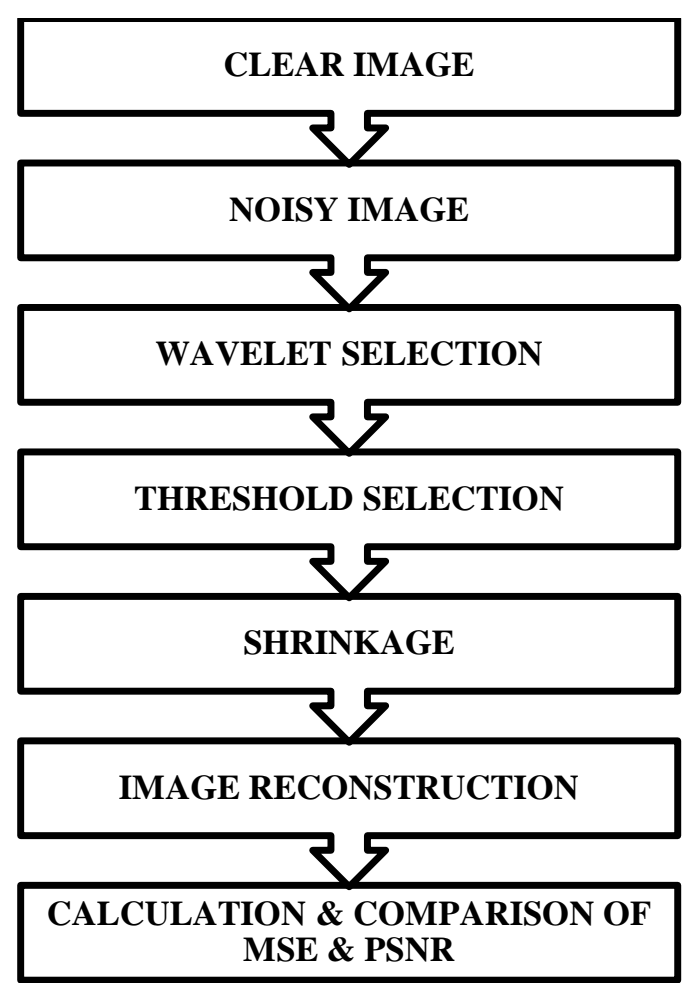

Figure 3: Methodology of Proposed work

Fig. 3 gives the methodolgy adopted for concerned research work in which the first step is to add gaussian noise in selected image after that a particular wavelet is selected followed by selection of threshold level for noise shrinkage and then the empirical parameters are calculted and compare for various selections. 


\section{EXPERIMENTAL RESULTS AND DISCUSSION}

The following images show the original image, recovered image after the denoising has been done by the hard \& soft thresholding using different wavelets. Comparison of MSE and PSNR of Different test images corrupted by Gaussian Noise has been done

Figures 4,5 and 6 represent the selected set of medical images and required processing on same for denoising and Tables1,2,3,4 and 5 give the calculation results of performance metrics for Haar, Dabechies \& Symlet wavelet with the hard \& soft thresholding. various terms used above can be described as:

Hard MSE = Mean square error of original image and reconstructed image.

Soft MSE = Mean square error of original image and reconstructed image.

Hard PSNR= Peak signal to noise ratio of original image and reconstructed image.

Soft PSNR= Peak signal to noise ratio of original image and reconstructed image.

Db2= Daubechies Wavelet

Sym4=Symlet Wavelet

We have calculated \& compare the value of MSE \& PSNR for hard \& soft thresholding for the three wavelets Haar, Daubechies \& Symlet and it has been observed that Symlet wavelet is the most efficient among all the three wavelets as its PSNR Value is the highest among all other wavelets at a particular level of Gaussian noise for all the particular images. The process has been repeated for three test images Brain Tumour Lung and Ultrasound images with noise variance of 0.01,0.02,0.03 and 0.04, it has been observed from Table 1,2 and 3 that all results are different for different images. All the three wavelets have been observed and analysed by implementing in MATLAB.
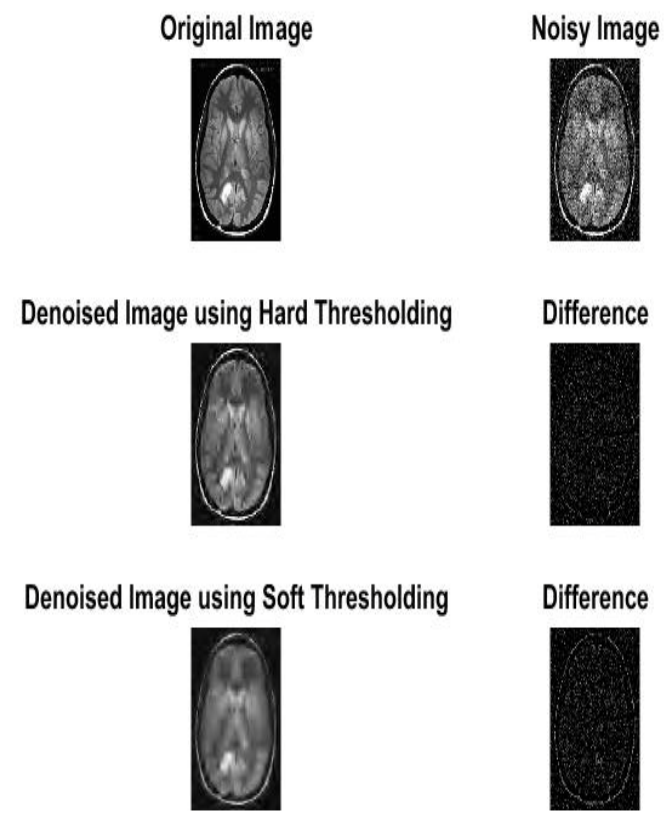

To estimate the efficiency of any image denoising method there are wo commonly used matrics i.e. are MeanSquared Error and Peak Signal-to-Noise Ratio. The meansquared error (MSE) between two images $i(x, y)$ (original image) and $\mathrm{i}^{\wedge}(\mathrm{x}, \mathrm{y})$ (reconstructed image) of $\mathrm{MN}$ dimensions

$\mathrm{MSE}=\frac{1}{M N} \sum_{\mathrm{y}=1}^{\mathrm{M}} \sum_{\mathrm{x}=1}^{\mathrm{N}}\left[i(x, y)-\mathrm{i}^{\wedge}(\mathrm{x}, \mathrm{y})\right]^{2}$

But the problem with mean-squared error is its dependency on scaling of image intensity scaling. The problem of MSE is avoided by PSNR which is a measure for comparing the denoising results of same image.

PSNR $=20 * \log 10(255 / \operatorname{sqrt}($ MSE $))$

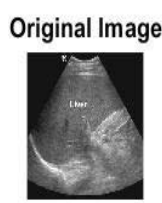

Denoised Image using Hard Thresholding

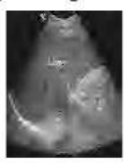

Denoised Image using Soft Thresholding

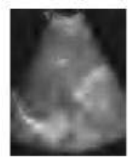

Noisy Image

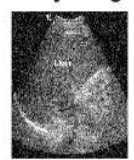

Difference

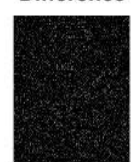

Difference

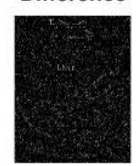

Figure 5: Liver image and respective wavelet processing on the same for denoising
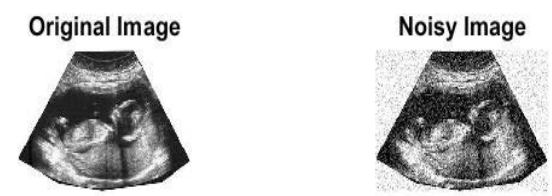

Denoised Image using Hard Thresholding

Difference
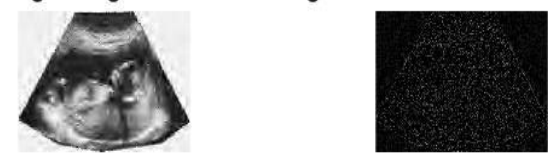

Denoised Image using Soft Thresholding

Difference
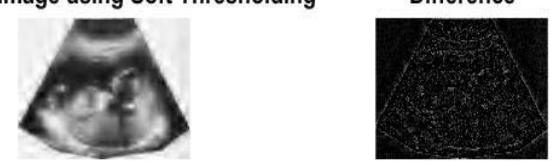

Figure 6: Ultrasound image and respective wavelet processing on the same for denoising 
Table1: Calculation of PSNR \& MSE values for Haar Wavelet

\begin{tabular}{|c|c|c|c|c|}
\hline $\begin{array}{l}\text { Image } \\
\text { Name }\end{array}$ & $\begin{array}{c}\text { Thersholding } \\
\text { Method }\end{array}$ & $\begin{array}{c}\text { Noise } \\
\text { Variance }\end{array}$ & PSNR & MSE \\
\hline \multirow{8}{*}{$\begin{array}{l}\text { Brain } \\
\text { Tumor } \\
\text { Image }\end{array}$} & \multirow[t]{4}{*}{ Hard } & 0.01 & 76.8162 & 0.0054 \\
\hline & & 0.02 & 69.0217 & 0.0081 \\
\hline & & 0.03 & 67.9914 & 0.0103 \\
\hline & & 0.04 & 67.1562 & 0.0125 \\
\hline & \multirow[t]{4}{*}{ Soft } & 0.01 & 68.3392 & 0.0095 \\
\hline & & 0.02 & 67.0439 & 0.0128 \\
\hline & & 0.03 & 66.2696 & 0.0154 \\
\hline & & 0.04 & 65.6851 & 0.0176 \\
\hline \multirow{8}{*}{$\begin{array}{l}\text { Liver } \\
\text { Image }\end{array}$} & \multirow[t]{4}{*}{ Hard } & 0.01 & 72.8533 & 0.0034 \\
\hline & & 0.02 & 71.7251 & 0.0044 \\
\hline & & 0.03 & 70.8558 & 0.0053 \\
\hline & & 0.04 & 70.3742 & 0.0060 \\
\hline & \multirow[t]{4}{*}{ Soft } & 0.01 & 71.4681 & 0.0046 \\
\hline & & 0.02 & 70.6729 & 0.0056 \\
\hline & & 0.03 & 70.0325 & 0.0065 \\
\hline & & 0.04 & 69.6221 & 0.0071 \\
\hline \multirow{8}{*}{$\begin{array}{c}\text { Ultra } \\
\text { sound } \\
\text { Image }\end{array}$} & \multirow[t]{4}{*}{ Hard } & 0.01 & 69.1907 & 0.0066 \\
\hline & & 0.02 & 68.2921 & 0.0094 \\
\hline & & 0.03 & 67.4204 & 0.0118 \\
\hline & & 0.04 & 66.7249 & 0.0218 \\
\hline & \multirow[t]{4}{*}{ Soft } & 0.01 & 67.5096 & 0.0116 \\
\hline & & 0.02 & 66.1173 & 0.0129 \\
\hline & & 0.03 & 65.7882 & 0.0181 \\
\hline & & 0.04 & 64.8200 & 0.0214 \\
\hline
\end{tabular}

Table2: Calculation of PSNR \& MSE values for Symlet Wavelet

\begin{tabular}{|c|c|c|c|c|}
\hline $\begin{array}{l}\text { Image } \\
\text { Name }\end{array}$ & $\begin{array}{l}\text { Thersholdi } \\
\text { ng Method }\end{array}$ & $\begin{array}{c}\text { Noise } \\
\text { Variance }\end{array}$ & PSNR & MSE \\
\hline \multirow{8}{*}{$\begin{array}{l}\text { Brain } \\
\text { Tumor } \\
\text { Image }\end{array}$} & \multirow[t]{4}{*}{ Hard } & 0.01 & 71.6421 & 0.0045 \\
\hline & & 0.02 & 69.7743 & 0.0068 \\
\hline & & 0.03 & 68.8225 & 0.0095 \\
\hline & & 0.04 & 67.9743 & 0.0104 \\
\hline & \multirow[t]{4}{*}{ Soft } & 0.01 & 69.1236 & 0.0080 \\
\hline & & 0.02 & 67.6879 & 0.0111 \\
\hline & & 0.03 & 66.9278 & 0.0132 \\
\hline & & 0.04 & 66.3895 & 0.0152 \\
\hline \multirow{8}{*}{$\begin{array}{l}\text { Liver } \\
\text { Image }\end{array}$} & \multirow[t]{4}{*}{ Hard } & 0.01 & 73.4093 & 0.0030 \\
\hline & & 0.02 & 72.4619 & 0.0037 \\
\hline & & 0.03 & 71.7000 & 0.0044 \\
\hline & & 0.04 & 71.1333 & 0.0050 \\
\hline & \multirow[t]{4}{*}{ Soft } & 0.01 & 72.2098 & 0.0039 \\
\hline & & 0.02 & 71.6119 & 0.0045 \\
\hline & & 0.03 & 71.0290 & 0.0051 \\
\hline & & 0.04 & 70.6023 & 0.0057 \\
\hline \multirow{8}{*}{$\begin{array}{l}\text { Ultra } \\
\text { sound } \\
\text { Image }\end{array}$} & \multirow[t]{4}{*}{ Hard } & 0.01 & 70.1404 & 0.0063 \\
\hline & & 0.02 & 68.7152 & 0.0087 \\
\hline & & 0.03 & 67.9170 & 0.0105 \\
\hline & & 0.04 & 67.2984 & 0.0121 \\
\hline & \multirow[t]{4}{*}{ Soft } & 0.01 & 68.0041 & 0.0103 \\
\hline & & 0.02 & 66.8456 & 0.0135 \\
\hline & & 0.03 & 66.1187 & 0.0159 \\
\hline & & 0.04 & 65.5869 & 0.0180 \\
\hline
\end{tabular}

Table3: Calculation of PSNR \& MSE values for Daubechies(db3)Wavelet

\begin{tabular}{|c|c|c|c|c|}
\hline $\begin{array}{l}\text { Image } \\
\text { Name }\end{array}$ & $\begin{array}{c}\text { Thersholdin } \\
\text { g Method }\end{array}$ & $\begin{array}{c}\text { Noise } \\
\text { Variance }\end{array}$ & PSNR & MSE \\
\hline \multirow{8}{*}{$\begin{array}{l}\text { Brain } \\
\text { Tumor } \\
\text { Image }\end{array}$} & \multirow[t]{4}{*}{ Hard } & 0.01 & 71.7408 & 0.0044 \\
\hline & & 0.02 & 70.1246 & 0.0063 \\
\hline & & 0.03 & 69.0583 & 0.0081 \\
\hline & & 0.04 & 68.2663 & 0.0097 \\
\hline & \multirow[t]{4}{*}{ Soft } & 0.01 & 69.3009 & 0.0076 \\
\hline & & 0.02 & 67.9323 & 0.0105 \\
\hline & & 0.03 & 67.1685 & 0.0127 \\
\hline & & 0.04 & 66.4897 & 0.0146 \\
\hline \multirow{8}{*}{$\begin{array}{l}\text { Liver } \\
\text { Image }\end{array}$} & \multirow[t]{4}{*}{ Hard } & 0.01 & 73.4614 & 0.0029 \\
\hline & & 0.02 & 72.3924 & 0.0037 \\
\hline & & 0.03 & 71.8760 & 0.0042 \\
\hline & & 0.04 & 71.1778 & 0.0050 \\
\hline & \multirow[t]{4}{*}{ Soft } & 0.01 & 72.3269 & 0.0038 \\
\hline & & 0.02 & 71.5351 & 0.0046 \\
\hline & & 0.03 & 71.1166 & 0.0050 \\
\hline & & 0.04 & 70.6366 & 0.0056 \\
\hline \multirow{8}{*}{$\begin{array}{l}\text { Ultra } \\
\text { sound } \\
\text { Image }\end{array}$} & \multirow[t]{4}{*}{ Hard } & 0.01 & 70.3793 & 0.0060 \\
\hline & & 0.02 & 68.9615 & 0.0083 \\
\hline & & 0.03 & 68.0813 & 0.0101 \\
\hline & & 0.04 & 67.4168 & 0.0118 \\
\hline & \multirow[t]{4}{*}{ Soft } & 0.01 & 68.3527 & 0.0095 \\
\hline & & 0.02 & 67.1321 & 0.0126 \\
\hline & & 0.03 & 66.4293 & 0.0148 \\
\hline & & 0.04 & 65.8672 & 0.0168 \\
\hline
\end{tabular}

\section{FUTURE SCOPE}

The statistical quantitative metrics have been calculated on a set of medical images of different resolution and work is being done to remove gaussian noise of the images and future plan is to make it valuable for every noise model and for different size of image.

\section{REFERENCES}

[1] Donoho D.L., and Johnstone I. M., 1994. Ideal spatial adaptationvia wavelet shrinkage Biometrika, vol. 81, pp. 425455

[2] Mallat S, Hwang W L. Singularity detection andprocessing with Wavelets [J]. IEEE Transactions on Information Theory, 1992, 38 (2) : 617-643.

[3] Image Denoising via Wavelet-Domain Spatially Adaptive FIR Wiener Filtering Zhang, H.; Nosratinia, A.; Wells, R.O., Jr.; Acoustics, Speech, and Signal Processing, 2000. ICASSP '00. Proceedings. 2000 IEEE International Conference on , Volume: 6 , 5-9 June 2000 Pages:2179 - 2182 vol.4

[4] R. C. Gonzalez, R. E. Woods, "Digital Image Processing using Matlab”, 2nd ed., Prentice Hall.

[5] A K Jain, 'Fundamentals of Digital Image Processing', Prentice Hall, NJ, 1990.

[6] Ingrid Daubechies “Ten lectures on wavelets” Philadelphia, PA: SLAM, 1992. 
[7] Raghuram Rangarajan, Ramji Venkataramanan and Siddharth Shah, Image Denoising Using Wavelets, December 16, 2002.

[8] Pawan Patidar, Manoj Gupta, Sumit Srivastava, AshokKumar Nagawat,“ Image De-noising by Various Filters for Different Noise”, International Journal of Computer Applications, November 2010, Vol.9, No.4,pp. 0975-887.
[9] Coifman, R. R., Meyer, Y., and Wickerhauser, M. V.,Wavelet Analysis and signal processing in Wavelets and their applications, Ruskai, B., Ed. Boston: Jones and Barlett, pp. 153178, 1992.

[10] Portilla, Javier, et al. "Image denoising using scale mixtures of Gaussians in the wavelet domain." Image Processing, IEEE Transactions on 12.11 (2003): 1338-1351. 